\title{
Preliminary Results on the Empirical Applicability of the Tsallis Distribution in Elastic Hadron Scattering
}

\author{
D. A. Fagundes, M. J. Menon, P. V. R. G. Silva \\ Universidade Estadual de Campinas - UNICAMP \\ Instituto de Física Gleb Wataghin \\ 13083-859 Campinas, SP, Brazil \\ fagundes@ifi.unicamp.br, \\ menon@ifi.unicamp.br, precchia@ifi.unicamp.br
}

\begin{abstract}
We show that the proton-proton elastic differential cross section data at dip position and beyond can be quite well described by a parametrization based on the Tsallis distribution, with only five free fit parameters. Extrapolation of the results obtained at $7 \mathrm{TeV}$ to large momentum transfer, suggests that hadrons may not behave as a black-disk at the asymptotic energy region.

PACS numbers: 13.85.-t, 13.85.Dz

Keywords: Hadron-induced high-energy interactions, Elastic scattering
\end{abstract}

\section{Contribution to XII Hadron Physics, Bento Gonçalves - RS, Brazil, 22-27th April 2012} To be published in AIP Proc. Conf.

The theoretical description of the elastic hadron-hadron differential cross section still constitutes a hard challenge for QCD. Experimental information available on proton-proton $(p p)$ scattering at $19-60 \mathrm{GeV}$ comprises eleven to twelve decades of experimental data, which turns out very difficult any phenomenological [1] or even empirical [2] description of this quantity. Moreover, recent results obtained by the TOTEM Collaboration at $7 \mathrm{TeV}$ have indicated that representative-model extrapolations to this energy are not consistent with the bulk of the experimental information, in special at large values of the momentum transfer 3 , 4].

On the other hand, the Tsallis distribution [5] has been widely used in the description of the transverse momentum spectra of identified particles [6 [8]. A striking feature is the fact that these spectra have similar shapes as those characteristic of the elastic differential cross sections at and beyond the dip position. Based on this similarity, we have developed an empirical analysis of the $p p$ differential cross section data with a parametrization based on the Tsallis distribution for the intermediate and high momentum transfer squared regions, together with two exponential functions for the low momentum region (the diffraction peak).

Different forms of the Tsallis distribution [5] have been used in the literature and several fundamental aspects are discussed, for example, in [6 8] and references therein. For our purpose we shall consider the version that has been used by the experimental collaborations (STAR, PHENIX, ALICE, ATLAS and CMS), which is expressed by

$$
\frac{d^{2} N}{d p_{T} d y}=\frac{d N}{d y} \frac{(n-1)(n-2)}{n T\left[n T+m_{0}(n-2)\right]} p_{T}\left[1+\frac{\sqrt{p_{T}^{2}+m_{0}^{2}}-m_{0}}{n T}\right]^{-n},
$$

where $p_{T}$ is the transverse momentum, $d N / d y$ the yield per unity rapidity $y, m_{0}$ the fixed hadron mass and $n$ and $T$ are shape parameters.

The main ingredient in this communication is to show that an analytical parametrization based on the above distribution can give quite good descriptions of the $p p$ differential cross section data at and beyond the dip position. However, in order to get a global description of the available data, an instrumental sum of two exponential are also considered for the region below the dip position (the diffraction peak region). For a matter of notation in a strictly empirical context, we shall denote these two forms by the regions they are intended for, namely: Diffraction Peak $(D P)$ contribution and beyond the Diffraction Peak (bDP) contribution. Specifically, we express the differential cross section in terms of the momentum transfer squared, $q^{2}$, as a sum of two terms,

$$
\frac{d \sigma}{d q^{2}}=\left.\frac{d \sigma}{d q^{2}}\right|_{D P}+\left.\frac{d \sigma}{d q^{2}}\right|_{b D P}
$$

The first one is parametrized as

$$
\left.\frac{d \sigma}{d q^{2}}\right|_{D P}=a_{1} e^{-b_{1} q^{2}}+a_{2} e^{-b_{2} q^{2}}
$$



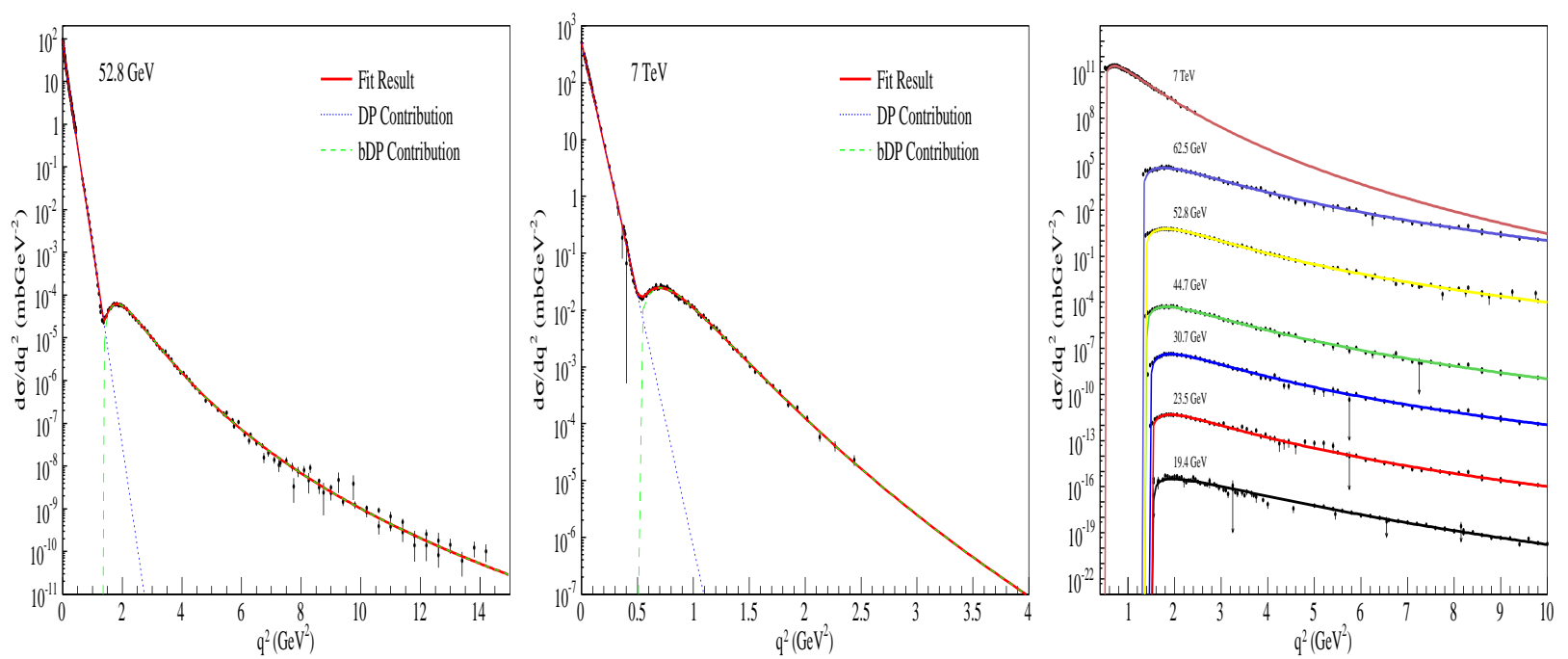

FIG. 1: Left and center: Typical fit results and contributions to the differential cross section from the Diffraction Peak (DP) parametrization (3) and beyond the Diffraction Peak (bDP) parametrization (4), together with the experimental data $(52.8 \mathrm{GeV}$ and $27.4 \mathrm{GeV})$ and extracted points $(7 \mathrm{TeV})$. Right: All results at the dip position and beyond with the bDP contribution, Eq. (4). Curves and data have been multiplied by factors of $10^{ \pm 2}$

where $a_{i}, b_{i}, i=1,2$ are free fit parameters. The second one is based on the Tsallis distribution shifted from the origin,

$$
\left.\frac{d \sigma}{d q^{2}}\right|_{b D P}=\alpha \frac{(\gamma-1)(\gamma-2)}{\gamma \beta[\gamma \beta+\delta(\gamma-2)]}\left[q^{2}-q_{0}^{2}\right]\left[1+\frac{\sqrt{\left[q^{2}-q_{0}^{2}\right]^{2}+\delta^{2}}-\delta}{\gamma \beta}\right]^{-\gamma},
$$

where $\alpha, \beta, \gamma, \delta$ and $q_{0}^{2}$ are free fit parameters.

As in previous empirical investigation [2], we shall base our analysis on six sets of $p p$ elastic scattering data, at $\sqrt{s}=19.4 \mathrm{GeV}, 23.5,30.7,44.7,52.8$ and $62.5 \mathrm{GeV}$. In addition, we make use of the empirical result that at the ISR energy region $(23.5-62.5 \mathrm{GeV})$ the differential cross section data above $q^{2} \sim 4 \mathrm{GeV}^{2}$ do not depend on the energy. This fact allows the inclusion in each set (ISR) the data at $27.4 \mathrm{GeV}$ (from Fermilab), covering the large momentum transfer region: $5.5 \leq q^{2} \leq 14.2 \mathrm{GeV}^{2}$ (see Ávila and Menon in [2] for a quantitative discussion on this respect). Only the statistical uncertainties of the experimental data are taken into account. Here, we shall also consider the new results on elastic $p p$ scattering at $7 \mathrm{TeV}$, recently obtained by the TOTEM Collaboration at the LHC. However, since the data points (numerical values) are not yet available in published format, we have used the applicative Plot Digitizer to extract the points and uncertainties from the plots in [3] and [4]. The data reductions have been performed with the objects of the class TMinuit of ROOT Framework. First, the two regions have been treated separately in order to infer start values of the parameters in a global final fit and in that case we have included in the fit code a step-function of $q^{2}-q_{0}^{2}$, before the bDP parametrization (4). Typical fit results (curves) are presented in Figure 1. The values of the fit parameters at $7 \mathrm{TeV}$ are: $a_{1}=505.4 \pm 1.4 \mathrm{mbGeV}^{-2}, b_{1}=20.429 \pm 0.029$ $\mathrm{GeV}^{-2}, \alpha=1.1321 \pm 0.0094 \times 10^{-2} \mathrm{mb}, \beta=0.1339 \pm 0.0056 \mathrm{GeV}^{2}, \gamma=19.6 \pm 3.1, \delta=0.216 \pm 0.029$ $\mathrm{GeV}^{2}$ and $q_{0}^{2}=0.5015 \pm 0.0029 \mathrm{GeV}^{2}\left(\chi^{2} / \mathrm{DOF}=1.65\right.$ for $\left.115 \mathrm{DOF}\right)$.

We conclude that parametrization (2-4) leads to good descriptions of all the $p p$ differential cross section data available at $\sqrt{s} \geq 19.4 \mathrm{GeV}$. A novel result concerns the monotonic decrease of the differential cross section (without any oscillation) at the deep elastic scattering region, $q^{2}$ above $\sim 4 \mathrm{GeV}^{2}[9]$, even at $7 \mathrm{TeV}$ (where the slope, with a small positive curvature, is nearly two times the value observed at the ISR region, as illustrated in Figure 1, right). Therefore, this pattern suggests a scenario in disagreement with the black-disk as an asymptotic limit. Another novel aspect, concerns the suggestion of a possible nonextensive thermal statistics interpretation of the dip-bump and deep elastic scattering regions. Further tests and investigation are in course. 


\section{Acknowledgments}

We are thankful to J. Takahashi and D.D. Chinellato for discussions. Research supported by FAPESP (Contracts Nos. 11/15016-4, 11/00505-0, 09/50180-0).

[1] J. Kašpar, V. Kundrát, M. Lokajíček and J. Procházka, Nucl. Phys. B 843, 84 (2011); R. Fiore et al., Int. J. Mod. Phys. A 24, 2551 (2009); G. Matthiae, Rep. Prog. Phys. 57, 743 (1994).

[2] D. A. Fagundes and M. J. Menon, Int. J. Mod. Phys. A 26, 3219 (2011); D. A. Fagundes, M. J. Menon and G. L. P. Silva, Eur. Phys. J. C 71, 1637 (2011); R. F. Ávila, and M. J. Menon, Eur. Phys. J. C 54, 555 (2008); P. A. S. Carvalho, A. F. Martini and M. J. Menon, Eur. Phys. J. C 39, 359 (2005); P. A. S. Carvalho and M. J. Menon, Phys. Rev. D 56, 7321 (1997).

[3] G. Antchev et al. (TOTEM Collaboration), Europhys. Lett. 9541001 (2011)

[4] G. Antchev et al. (TOTEM Collaboration), Europhys. Lett. 9621002 (2011)

[5] C. Tsallis, J. Statist. Phys. 52, 479 (1988).

[6] G. Wilk and Z. Włodarczyk, Eur. Phys. J. A 40, 299 (2009); Phys. Rev. C 79, 54903 (2009).

[7] J. Cleymans and D. Worku, arXiv:1203.4343 [hep-ph]; J. Phys. G 39025006 (2012).

[8] B. De, G. Sau, S. K. Biswas, S. Bhattacharyya and P. Guptaron, Int. J. Mod. Phys. A 25, 1239 (2010).

[9] M. M. Islam, J. Kašpar and R. J. Luddy Mod. Phys. Lett. A 24, 485 (2009). 